\title{
Clinical Utility of KidneyIntelX in Early Stages of Diabetic Kidney Disease in the CANVAS Trial
}

\author{
David Lam ${ }^{\mathrm{a}}$ Girish N. Nadkarni ${ }^{\mathrm{b}}$ Gohar Mosoyan $^{\mathrm{b}}$ Bruce Nealc \\ Kenneth W. Mahaffey ${ }^{d}$ Norman Rosenthal $^{e}$ Michael K. Hansen ${ }^{e}$ \\ Hiddo J.L. Heerspink ${ }^{f}$ Fergus Fleming ${ }^{g}$ Steven G. Coca ${ }^{b}$ \\ aDivision of Endocrinology, Icahn School of Medicine at Mount Sinai, New York, NY, USA; 'bivision of Nephrology, \\ Icahn School of Medicine at Mount Sinai, New York, NY, USA; 'The George Institute for Global Health, UNSW Sydney, \\ Sydney, NSW, Australia; 'Department of Medicine, Stanford Center for Clinical Research, Stanford University School \\ of Medicine, Stanford, CA, USA; 'Janssen Research \& Development, LLC, Spring House, PA, USA; fDepartment of \\ Clinical Pharmacy and Pharmacology, University of Groningen, Groningen, The Netherlands; ${ }^{9}$ Renalytix Al, PLC, \\ New York, NY, USA
}

\section{Keywords}

Biomarkers · Diabetic nephropathy · Prognosis · Response to therapy

\begin{abstract}
Introduction: KidneyIntelX is a composite risk score, incorporating biomarkers and clinical variables for predicting progression of diabetic kidney disease (DKD). The utility of this score in the context of sodium glucose co-transporter 2 inhibitors and how changes in the risk score associate with future kidney outcomes are unknown. Methods: We measured soluble tumor necrosis factor receptor (TNFR)-1, soluble TNFR-2, and kidney injury molecule 1 on banked samples from CANagliflozin cardioVascular Assessment Study (CANVAS) trial participants with baseline DKD (estimated glomerular filtration rate $\left[\right.$ eGFR] $30-59 \mathrm{~mL} / \mathrm{min} / 1.73 \mathrm{~m}^{2}$ or urine albumin-to-creatinine ratio [UACR] $\geq 30 \mathrm{mg} / \mathrm{g}$ ) and generated KidneylntelX risk scores at baseline and years 1, 3, and 6. We assessed the association of baseline and changes in KidneyIntelX with subsequent DKD progression (composite outcome of an eGFR decline of $\geq 5 \mathrm{~mL} / \mathrm{min} /$ year [using the 6-week eGFR as the baseline in the canagliflozin group],
\end{abstract}

karger@karger.com www.karger.com/ajn

Karger!
(C) 2022 The Author(s)

Published by S. Karger AG, Basel

This is an Open Access article licensed under the Creative Common Attribution-NonCommercial-4.0 International License (CC BY-NC) (http://www.karger.com/Services/OpenAccessLicense), applicable to the online version of the article only. Usage and distribution for commercial purposes requires written permission. $\geq 40 \%$ sustained decline in the eGFR, or kidney failure). $\boldsymbol{R e}$ sults: We included 1,325 CANVAS participants with concurrent DKD and available baseline plasma samples (mean eGFR $65 \mathrm{~mL} / \mathrm{min} / 1.73 \mathrm{~m}^{2}$ and median UACR $56 \mathrm{mg} / \mathrm{g}$ ). During a mean follow-up of 5.6 years, 131 participants (9.9\%) experienced the composite kidney outcome. Using risk cutoffs from prior validation studies, KidneylntelX stratified patients to low- (42\%), intermediate- (44\%), and high-risk (15\%) strata with cumulative incidence for the outcome of $3 \%$, $11 \%$, and $26 \%$ (risk ratio $8.4 ; 95 \%$ confidence interval $[\mathrm{Cl}]: 5.0$, 14.2) for the high-risk versus low-risk groups. The differences in eGFR slopes for canagliflozin versus placebo were 0.66 , 1.52 , and $2.16 \mathrm{~mL} / \mathrm{min} / 1.73 \mathrm{~m}^{2}$ in low, intermediate, and high KidneylntelX risk strata, respectively. KidneylntelX risk scores declined by $5.4 \%(95 \% \mathrm{Cl}:-6.9,-3.9)$ in the canagliflozin arm at year 1 versus an increase of $6.3 \%$ (95\% Cl: 3.8, $8.7)$ in the placebo arm $(p<0.001)$. Changes in the KidneylntelX score at year 1 were associated with future risk of the composite outcome (odds ratio per 10 unit decrease 0.80; $95 \% \mathrm{Cl}: 0.77,0.83 ; p<0.001)$ after accounting for the treatment arm, without evidence of effect modification by the baseline KidneylntelX risk stratum or by the treatment arm. Conclusions: KidneylntelX successfully risk-stratified a large 
multinational external cohort for progression of DKD, and greater numerical differences in the eGFR slope for canagliflozin versus placebo were observed in those with higher baseline KidneyIntelX scores. Canagliflozin treatment reduced KidneyIntelX risk scores over time and changes in the KidneyIntelX score from baseline to 1 year associated with future risk of DKD progression, independent of the baseline risk score and treatment arm.

2022 The Author(s)

Published by S. Karger AG, Basel

\section{Introduction}

Diabetic kidney disease (DKD) affects $25-40 \%$ of individuals with type 2 diabetes. Diabetes is the leading cause of end-stage kidney disease in the USA. However, only a fraction of individuals with DKD will experience progression to advanced stages of disease and end-stage kidney disease. Better tools for risk stratification are needed to identify those at highest risk of DKD progression. Several biomarkers that reflect the pathophysiologic processes implicated in disease progression, such as inflammation and kidney tubular injury, have been investigated and shown to be prognostic of future events. Three of the most studied biomarkers that have shown consistent association with future disease progression and provided independent information along with standard clinical variables, such as the estimated glomerular filtration rate (eGFR) or urine albumin-tocreatinine ratio (UACR), are tumor necrosis factor receptor (TNFR) 1, TNFR-2, and kidney injury molecule 1 (KIM-1) [1-5].

Sodium glucose co-transporter 2 inhibitors (SGLT2i) have demonstrated a consistent ability to reduce DKD progression [6-9]. In a post hoc analysis of the CANagliflozin cardioVascular Assessment Study (CANVAS), Sen et al. [10] demonstrated that baseline values of TNFR-1, TNFR-2, and KIM-1 were associated with kidney outcomes and that canagliflozin reduced the concentration of these 3 biomarkers compared to the placebo and that early changes in TNFR-1 and TNFR- 2 were independently associated with subsequent kidney events. However, the integration of information from 3 different biomarkers into clinical practice is challenging. KidneyIntelX, a new bioprognostic test, incorporates inputs from 3 biomarkers (TNFR-1, TNFR-2, and KIM-1) along with 7 standard clinical variables and provides a discrete continuous risk score (5-100) that is further divided into 3 categorical groups of low, intermediate, or high risk [11].
This article seeks to build upon the previous findings from validation studies of KidneyIntelX. Specifically, our goals were as follows: (1) to demonstrate that baseline KidneyIntelX assessment in the intended-use population provides accurate risk stratification for kidney outcomes in an independent, internationally recruited randomization control trial cohort (those with type 2 diabetes and DKD stages 1-3); (2) to assess the impact of canagliflozin versus placebo in patients within risk strata of KidneyIntelX risk; (3) to assess the impact of SGLT2i treatment on changes in longitudinal KidneyIntelX risk scores over time; and (4) to determine the added value of early longitudinal changes in KidneyIntelX for forecasting subsequent kidney outcomes (i.e., progressive DKD).

\section{Methods}

\section{Patients and the Study Design}

The CANVAS Program consisted of 2 multicenter, doubleblinded, placebo-controlled, randomized trials (CANVAS and CANVAS-R) done to assess the effect of canagliflozin on primarily cardiovascular events, kidney function, and safety outcomes in patients with type 2 diabetes who had a history of cardiovascular disease or multiple cardiovascular risk markers as previously described [12]. Blood and urine samples for exploratory biomarker research were only available from the CANVAS trial and have been used for the current study. The CANVAS trial enrolled 4,330 participants from 24 countries. Participants were randomly assigned using a central Web-based response system in a 1:1:1 ratio for treatment with canagliflozin $100 \mathrm{mg}$, canagliflozin $300 \mathrm{mg}$, or a matching placebo. Participants assigned to treatment with canagliflozin or the placebo were followed for a median of 6.1 years. All participants, care providers, trial staff, and outcome assessors were blinded to treatment allocation for the duration of the study. All participants provided written informed consent with a separate and optional informed consent for the collection of additional blood or urine samples for biomarker analysis. The CANVAS trial was conducted according to the principles of the Declaration of Helsinki and was registered with ClinicalTrials.gov (NCT01032629). The CANVAS trial was approved by an Ethics Committee at each participating site, and all participants provided written informed consent.

\section{KidneyIntelX Test}

The KidneyIntelX test predicts risk of the composite outcome of progressive decline in kidney function in individuals with type 2 diabetes and concurrent DKD within 5 years (i.e., eGFR $\geq 30-59.9 \mathrm{~mL} /$ $\mathrm{min} / 1.73 \mathrm{~m}^{2}$ [G3a and G3b] or those with an eGFR $\geq 60 \mathrm{~mL} / \mathrm{min} / 1.73$ $\mathrm{m}^{2}$ with an UACR $\geq 30 \mathrm{mg} / \mathrm{g}$ ). KidneyIntelX was evaluated in the subgroup of the CANVAS population that met the criteria for prevalent $\mathrm{DKD}$ at the time of enrollment. The KidneyIntelX test measures plasma TNFR-1, TNFR-2, and KIM-1 and combines these biomarker results with select clinical features using machine learning to predict any of the following as a composite event: (A) rapid kidney function decline (RKFD; eGFR decline of $\geq 5 \mathrm{~mL} / \mathrm{min} /$ year), (B) a sustained $40 \%$ decline of the eGFR, or (C) kidney failure defined as an eGFR $<15 \mathrm{~mL} /$ $\mathrm{min} / 1.73 \mathrm{~m}^{2}$ or need for dialysis or kidney transplantation [11].
Lam et al. 
Biomarker Assessment

Blood samples for biomarker determinations were obtained at baseline, 52, 156, and 312 weeks after randomization. Plasma TNFR-1, TNFR-2, and KIM-1 were measured using a high-performance electrochemiluminescence immunoassay on the Mesoscale SECTOR S 600 instrument in the Renalytix AI laboratory in New York, NY, USA. All biomarkers were measured between August 2019 and December 2019. Overall, intra-assay coefficients of variation were all $\leq 3.3 \%$, and inter-assay coefficients of variation were $<6.5 \%$ for all 3 biomarkers (online suppl. Table. 1 ; for all onlinesuppl.material,seewww.karger.com/doi/10.1159/000519920). All assays were performed by personnel blinded to clinical outcomes.

\section{Outcomes}

The inputs for the kidney endpoint incorporated the eGFR values and adjudicated kidney outcomes as utilized in prior analyses of CANVAS [13-15]. The composite kidney outcome for this post hoc analysis was defined as above and, as per previous studies, used to validate KidneyIntelX performance $[16,17]$. In CANVAS, eGFR measurements were available at baseline, at 6 weeks, and annually. Given the known hemodynamic effects of the SGLT2i on acute changes in the eGFR, the eGFR value at 6 weeks was used as the anchor/baseline to define RKFD and 40\% decline in eGFR elements of the composite for participants in the canagliflozin arm [13-15]. If the eGFR value met $\geq 40 \%$ decline from baseline only on the last eGFR obtained in the CANVAS protocol, then the event was adjudicated by a blinded adjudication committee to determine qualification as a valid event [13-15]. eGFR slopes were determined by linear mixed models.

\section{Statistical Analyses}

Continuous baseline variables with normal distributions were reported as means with standard deviations. Variables with skewed distributions were reported as median values with interquartile ranges. Categorical variables were reported as percentages.

KidneyIntelX risk scores were calculated (blinded to the outcome) on the 1,325 participants in both placebo and canagliflozin arms of CANVAS with DKD. KidneyIntelX includes baseline TNFR-1, TNFR-2, and KIM-1 levels; TNFR-2/TNFR-1 ratio; KIM-1/TNFR-1 ratio; eGFR; UACR; HbA1c; systolic blood pressure; liver function tests (aspartate aminotransferase); platelets; and serum calcium. Each participant was assigned a score proportional to the probability of experiencing the composite endpoint based on a continuous KidneyIntelX score between 5 and 100. As per the recent validation study, the KidneyIntelX categorical risk groups were as follows: low risk (score of 5-45), intermediate risk (score of 50-85), and high risk (score of $>85-100$ ) [11]. We determined the proportion of participants experiencing the composite kidney event within the categorical risk strata and used logistic regression to determine the relative odds of the endpoint for high versus low risk and intermediate risk score strata.

To provide context for clinical utility of the KidneyIntelX test, the proportion of participants who experienced the composite kidney outcome by KidneyIntelX risk strata were compared to the proportion as classified by their Kidney Disease Improving Global Outcomes (KDIGO) risk strata based on baseline eGFR and UACR values [6]. The odds ratios for high versus low KidneyIntelX risk groups were compared to KDIGO very high risk versus moderate risk using the $p$ value test for heterogeneity in a random-effects

KidneyIntelX Utility in Longitudinal

Therapy with SGLT2i weighted model. We also assessed the net reclassification index for KidneyIntelX risk strata versus KDIGO risk strata in those who did and did not ultimately experience the events.

We also explored the observed treatment effect of canagliflozin versus placebo on kidney outcomes determined categorically (composite kidney outcome) and continuously via mixed linear models to assess the effects on the chronic eGFR slope, using the 6-week eGFR in the canagliflozin arm to account for the acute hemodynamic effects of SGLT2i.

The effect of canagliflozin versus placebo on KidneyIntelX risk scores over time was assessed by calculating the difference of change in KidneyIntelX between treatment arms using repeated measures ANOVA models. Associations between the 1-year change in KidneyIntelX risk scores from baseline and the composite kidney outcome were assessed using logistic regression with a landmark approach. All kidney endpoints that occurred in the first year were excluded from the analysis. The models were adjusted for the treatment arm and, given the marked difference in the absolute incidence of the composite kidney outcome by baseline risk, were assessed and stratified by the baseline KidneyIntelX risk category. An interaction term incorporating the baseline KidneyIntelX risk stratum with randomized treatment assignment was also assessed for effect modification. Finally, we examined the proportion of participants who remained in the same risk strata at baseline to 1 year versus those who changed risk strata over time (overall and stratified by the treatment arm) to assess how recategorization of risk was associated with future kidney events. All analyses were performed in $\mathrm{R}$ version 3.5.1.

\section{Results}

\section{Study Population}

Of the 1,396 participants in the CANVAS trial with prevalent DKD, 1,325 had available blood samples at baseline, and 1,019 had samples available at baseline and at 1 year (shown in online suppl. Fig. 1). The mean age of the full study population was 64 years, where $32 \%$ were female, the mean eGFR was $65 \mathrm{~mL} / \mathrm{min} / 1.73 \mathrm{~m}^{2}$, and the median UACR was $56 \mathrm{mg} / \mathrm{g}$ (shown in Table 1). For the analysis of the association between 1-year changes in KidneyIntelX and the subsequent kidney outcome, 3 of the 1,016 participants were excluded since they experienced the time-to-event kidney outcome events ( $40 \%$ sustained decline in the eGFR or kidney failure) before year 1, leaving 1,013 available for the longitudinal analyses. Weights for the relative importance of features in the KidneyIntelX risk score are presented in online supplementary Figure 2 .

\section{Association between the Baseline KidneyIntelX Risk Score and the Composite Kidney Outcome}

During a mean follow-up of $6.2(5.8-7.3)$ years, 131 (9.9\%) of the 1,325 participants with baseline DKD ex- 
Table 1. Baseline characteristics of CANVAS participants with DKD and with baseline samples analyzed

\begin{tabular}{|c|c|}
\hline Age, median (Q1-Q3), years & $64(58-70)$ \\
\hline Female, $n(\%)$ & $444(31.8)$ \\
\hline \multicolumn{2}{|l|}{ Race, $n(\%)$} \\
\hline White & $1,127(81.3)$ \\
\hline African-American & 37 (2.6) \\
\hline Other & $232(16.6)$ \\
\hline CVD history, $n(\%)$ & $873(62.5)$ \\
\hline History of heart failure, $n(\%)$ & $228(16.3)$ \\
\hline Systolic BP, median (Q1-Q3), mm Hg & 138 (129-149) \\
\hline Follow-up time, median (Q1-Q3), months & $74(70-78)$ \\
\hline \multicolumn{2}{|l|}{ Laboratory characteristics } \\
\hline \multicolumn{2}{|l|}{ eGFR strata, $n(\%), \mathrm{mL} / \mathrm{min} / 1.73 \mathrm{~m}^{2}$} \\
\hline $30-44.9$ & $138(9.8)$ \\
\hline $45-59.9$ & $466(33.3)$ \\
\hline $60-89.9$ & $576(41.2)$ \\
\hline$\geq 90$ & $216(15.4)$ \\
\hline Baseline UACR, median (Q1-Q3), mg/g & $56(25-159)$ \\
\hline $\mathrm{UACR}<30 \mathrm{mg} / \mathrm{g}, n(\%)$ & $373(26.7)$ \\
\hline UACR 30-299 mg/g, n (\%) & 809 (57.9) \\
\hline UACR $\geq 300 \mathrm{mg} / \mathrm{g}, n(\%)$ & $210(15)$ \\
\hline Baseline HbA1c, median (Q1-Q3), \% & $8.2(7.5-8.9)$ \\
\hline \multicolumn{2}{|l|}{ Plasma biomarkers, median (Q1-Q3), pg/mL } \\
\hline TNFR-1 & $3,010(2,461-3,748)$ \\
\hline TNFR-2 & $11,320(9,030-14,077)$ \\
\hline KIM-1 & $149(98-249)$ \\
\hline \multicolumn{2}{|l|}{ Cumulative incidence of events, $n$ (\%) } \\
\hline eGFR slope $\geq 5 \mathrm{~mL} / \mathrm{min} / 1.73 \mathrm{~m}^{2} /$ year & $82(5.9)$ \\
\hline Sustained $40 \%$ decline in the eGFR or kidney failure & $95(6.8)$ \\
\hline Composite endpoint & $134(9.6)$ \\
\hline
\end{tabular}

perienced the composite kidney outcome. Using risk cutoffs from prior validation studies, the cumulative incidence of the composite kidney outcomes increased as KidneyIntelX risk scores increased (shown in Fig. 1). In terms of the KidneyIntelX risk strata, $41.7 \%$ of participants were classified as low risk, $43.8 \%$ were classified as intermediate risk, and $14.6 \%$ were classified as high risk. The corresponding incidence of the composite outcome was $3.1 \%, 10.9 \%$, and $26.4 \%$ for low, intermediate, and high risk, respectively, with an overall risk ratio of 8.4 (95\% confidence interval [CI]: 5.0, 14.2) for the high-risk versus low-risk groups. In the canagliflozin arm, the corresponding incidence was 3.6\%, $9.5 \%$, and $23.0 \%$, respectively, yielding a risk ratio of 6.0 (95\% CI: 3.2, 11.3) for the high-risk versus low-risk groups. In the placebo arm, the corresponding incidence was $2.1 \%, 13.3 \%$, and $32.4 \%$, yielding a risk ratio of 15.6 (95\% CI: 5.6, 43.6; shown in Table 2; Fig. 2). By way of illustration for how KidneyIntelX can be incorporated into clinical practice, we compared the risk categorization to the KDIGO categorization based on the eGFR and UACR, which stratified $69 \%, 23 \%$, and $8 \%$ of the DKD population into "moderately increased risk," "high risk," and "very high risk," with event rates of 7\%, $17 \%$, and $18 \%$ (relative risk [RR] of 2.5 [95\% CI: 1.8, 3.6] overall for "very high risk" vs. "moderately increased risk"; 2.3 [95\% CI: 1.3, 4.2] in the canagliflozin arm; and 3.4 [95\% CI: 1.5, 7.5] in the placebo arm). The net reclassification index (continuous) for KidneyIntelX risk strata versus KDIGO risk strata was 0.342 overall, 0.227 
Table 2. Associations of baseline KidneyIntelX and KDIGO risk strata with the composite kidney outcome

\begin{tabular}{|c|c|c|c|c|c|c|c|}
\hline Baseline risk & Risk category & \multicolumn{2}{|l|}{ overall } & \multicolumn{2}{|l|}{ canagliflozin } & \multicolumn{2}{|l|}{ placebo } \\
\hline KidneylntelX & Low risk & $17 / 552(3.1)$ & Ref & $13 / 342(3.8)$ & Ref & 4/193 (2.1) & Ref \\
\hline \multirow[t]{3}{*}{ KDIGO } & Moderate risk & $61 / 914(6.7)$ & Ref & $42 / 590(7.1)$ & Ref & $19 / 324(5.8)$ & Ref \\
\hline & High risk & $51 / 303(16.8)$ & $2.5(1.8,3.6)$ & $23 / 195(12.0)$ & $1.7(1.0,2.7)$ & $28 / 108$ (25.9) & $4.4(2.6,7.6)$ \\
\hline & Very high risk & 19/108 (17.6) & $2.6(1.6,4.2)$ & $12 / 73(16.4)$ & $2.3(1.3,4.2)$ & $7 / 35(20.0)$ & $3.4(1.5,7.5)$ \\
\hline
\end{tabular}

KDIGO, Kidney Disease Improving Global Outcomes; $\mathrm{Cl}$, confidence interval. ${ }^{\mathrm{a}}$ The risk ratio for intermediate risk or high risk versus low risk.

Fig. 1. Cumulative incidence of the composite kidney outcome by continuous KidneyIntelX risk scores. CI, confidence interval.

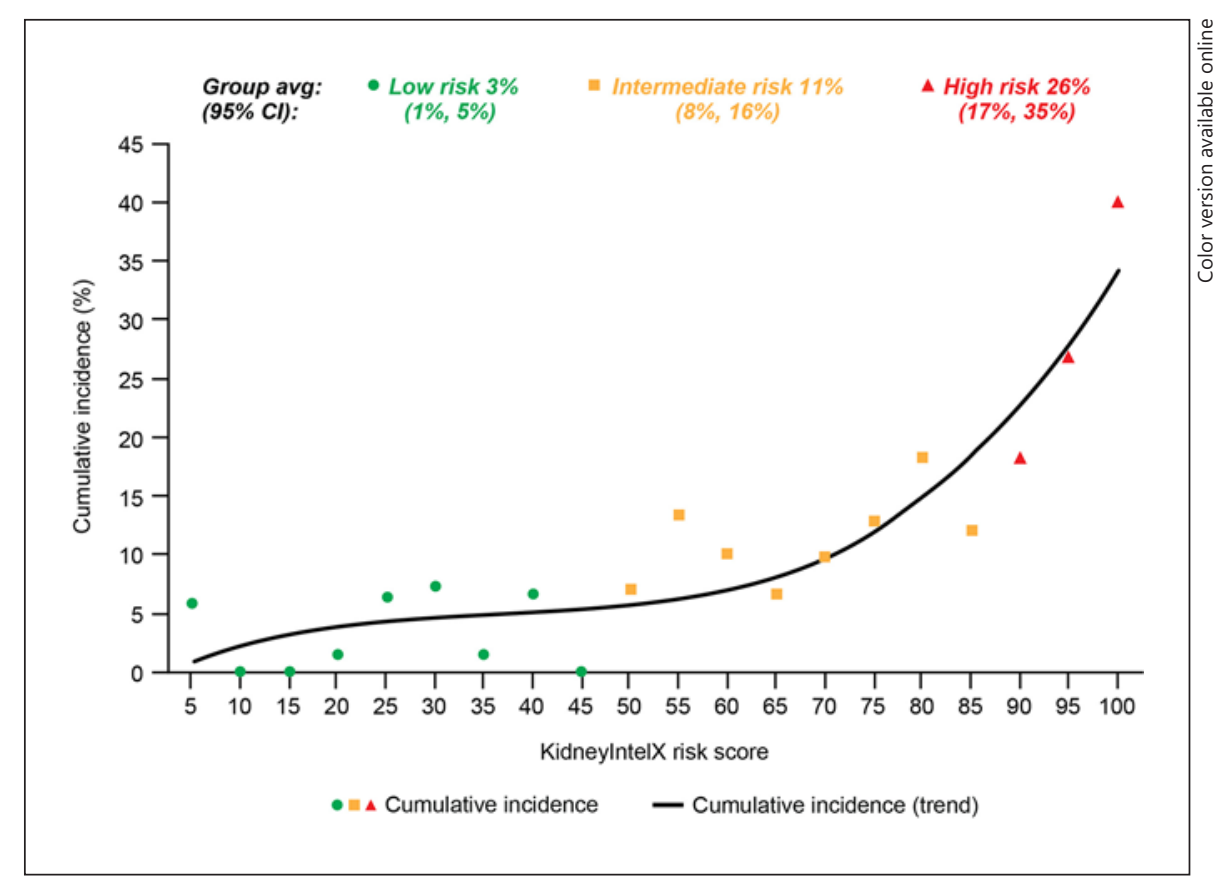

for those who developed the composite outcome, and 0.115 in those who did not experience the composite outcome.

\section{Effect of Canagliflozin on Kidney Outcomes}

(Composite Outcome and eGFR Slope) by Baseline

KidneyIntelX Risk Strata

In all participants with baseline biomarkers $(n=1,325)$, the composite kidney outcome occurred less frequently in the canagliflozin group (8.9\%) compared to the placebo group (11.6\%; RR for canagliflozin vs. placebo 0.70
[95\% CI: 0.49, 0.99]). In the KidneyIntelX low-risk stratum, the RR for the kidney outcome was 1.65 (95\% CI: $0.54,5.08)$ for canagliflozin versus placebo; in the KidneyIntelX intermediate-risk stratum, the RR was 0.59 (95\% CI: 0.36, 0.98); and in the KidneyIntelX high-risk stratum, the RR was 0.67 (95\% CI: $0.38,1.18$; $p$ for heterogeneity $=0.17$; shown in online suppl. Fig. 3 ). In the highrisk KidneyIntelX stratum, 31.4\% experienced the composite kidney outcome in the placebo arm and $23.5 \%$ in the canagliflozin arm (absolute risk reduction for canagliflozin of 7.9\%). 


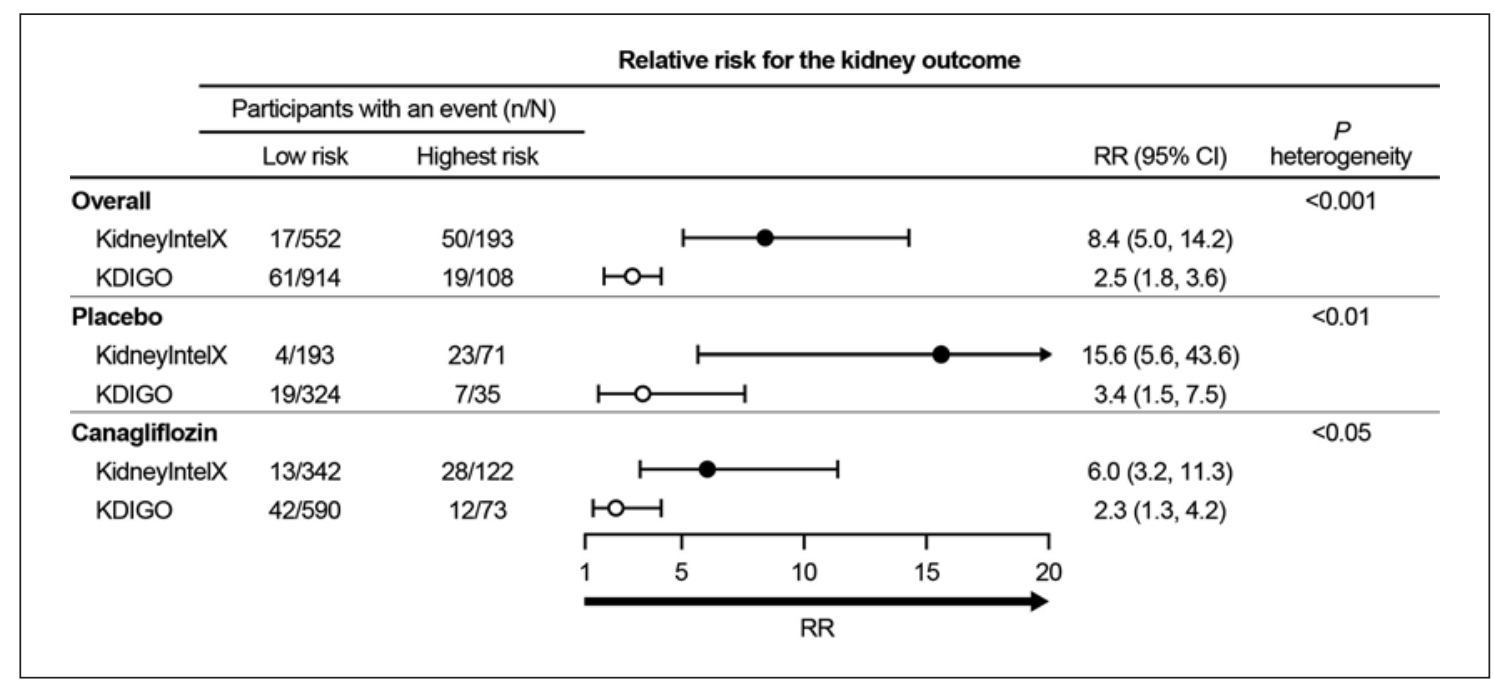

Fig. 2. Absolute and RR for kidney outcomes by KidneyIntelX versus KDIGO. $p$ value test for heterogeneity between the RR for KidneyIntelX versus KDIGO by the overall population and placebo and canagliflozin arms individually. RR, relative risk; CI, confidence interval; KDIGO, Kidney Disease Improving Global Outcomes.

We also examined the effect of canagliflozin versus placebo on chronic eGFR slopes by KidneyIntelX strata (shown in Fig. 3). The difference in the eGFR slope for canagliflozin versus placebo by KidneyIntelX risk strata was $0.66 \mathrm{~mL} / \mathrm{min} / 1.73 \mathrm{~m}^{2}$ in low risk, $1.52 \mathrm{~mL} / \mathrm{min} / 1.73$ $\mathrm{m}^{2}$ in intermediate risk, and $2.16 \mathrm{~mL} / \mathrm{min} / 1.73 \mathrm{~m}^{2}$ in high risk ( $p$ for interaction $=0.15$ ). The difference in the eGFR slope for canagliflozin versus placebo in the high-risk KidneyIntelX stratum $\left(2.16 \mathrm{~mL} / \mathrm{min} / 1.73 \mathrm{~m}^{2}\right)$ was of greater magnitude than the effect of canagliflozin versus placebo in the highest risk KDIGO stratum $(1.31 \mathrm{~mL} /$ $\min / 1.73 \mathrm{~m}^{2} ; p<0.001$; shown in Fig. 3 ).

\section{Effect of Canagliflozin on KidneyIntelX Risk Scores over Time}

Among the 1,013 participants with baseline and year 1 samples available, the continuous KidneyIntelX risk score increased from baseline to year 1 in the placebo group by $6.2 \%$ (95\% CI: $3.8,8.6)$ and decreased by $5.4 \%$ (95\% CI: $-6.9,-3.9)$ in those randomized to canagliflozin $(p<$ 0.001). This effect of canagliflozin on the KidneyIntelX risk score persisted over time until the end of the followup (shown in Fig. 4).

\section{Associations between Changes in KidneyIntelX and \\ Outcomes}

We examined the association of the change in KidneyIntelX from baseline to year 1 with the composite kidney outcome among the 1,013 participants with available samples and no event prior to year 1. Overall, stratified by the baseline KidneyIntelX score and adjusted for the treatment arm, each $10 \%$ reduction in KidneyIntelX risk was associated with a $20 \%$ lower risk of experiencing the composite kidney outcome (adjusted odds ratio per $10 \%$ reduction of 0.80 [95\% CI: $0.77,0.83$ ]; $p<0.001$ ). Although the median changes in KidneyIntelX varied markedly by baseline risk strata (shown in Fig. 5), there was no evidence of interaction for the association between the change in KidneyIntelX and the risk for the outcomes (each $10 \%$ reduction in KidneyIntelX was associated with an $18 \%, 21 \%$, and $18 \%$ reduction in the risk for the outcome by low, intermediate, and high baseline KidneyIntelX risk strata, respectively). In addition, there was no effect modification on the association between changes and outcomes by the treatment arm $\left(p_{\text {interaction }}=0.59\right)$. Cumulative incidence and the RR for movement from categories of KidneyIntelX risk from baseline to the follow-up are shown in online supplementary Figure 4.

\section{Discussion}

We have previously demonstrated that KidneyIntelX is prognostic for kidney disease progression in patients with type 2 diabetes and early-stage DKD in cohorts of patients recruited from outpatient settings into electronic health record-linked biobanks. This study further validates KidneyIntelX in the subgroup of corresponding
Lam et al. 
Fig. 3. Absolute effect of canagliflozin versus placebo on the eGFR slope by KidneyIntelX (a) and KDIGO risk strata (b). eGFR, estimated glomerular filtration rate; $\mathrm{CI}$, confidence interval; KDIGO, Kidney Disease Improving Global Outcomes.

Fig. 4. Changes in KidneyIntelX over time in canagliflozin versus placebo-treated participants.
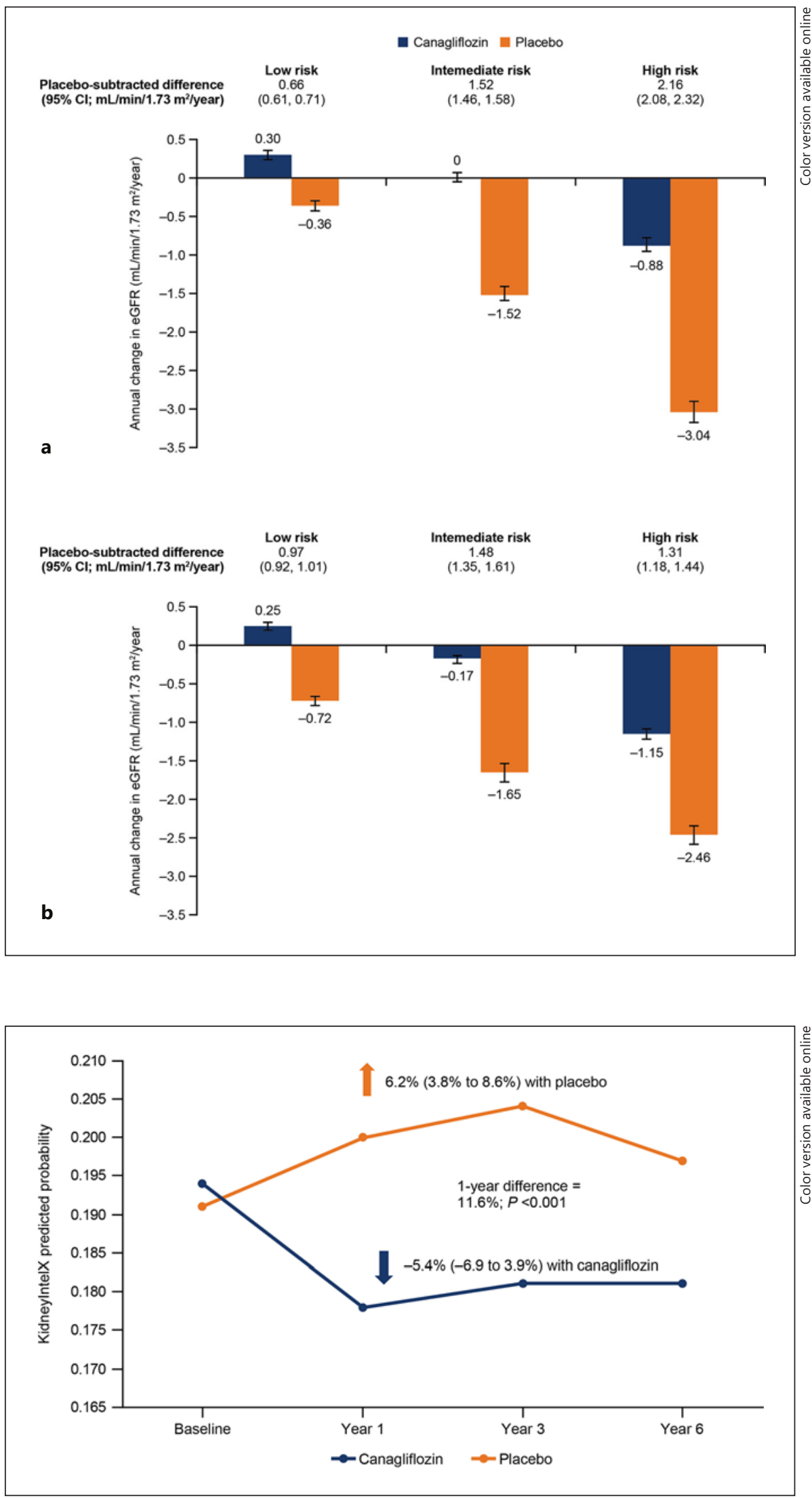

KidneyIntelX Utility in Longitudinal Therapy with SGLT2i
Am J Nephrol 2022;53:21-31

DOI: $10.1159 / 000519920$ 
Fig. 5. Change in KidneyIntelX and risk for composite kidney outcome. Tertiles of changes in KidneyIntelX risk scores from baseline to the follow-up, stratified by baseline KidneyIntelX risk stratum. Range of percent changes is shown for each stratum in each tertile.

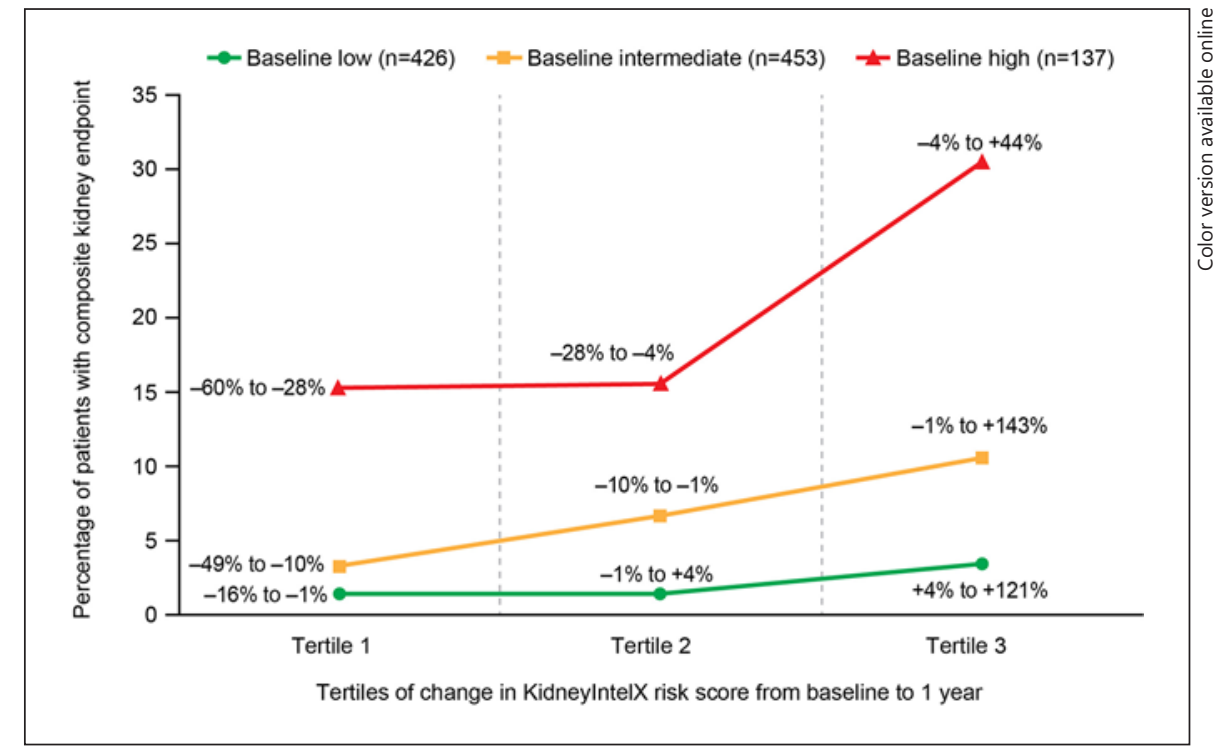

participants in a multinational clinical trial (CANVAS). The clinical trial design provided the framework to demonstrate a potential role of KidneyIntelX risk stratification and response to a novel therapeutic. First, the effects of the SGLT2i canagliflozin on the chronic eGFR slope were numerically greater in magnitude in participants who scored as high risk by KidneyIntelX at enrollment. Second, canagliflozin decreased KidneyIntelX risk scores over time compared to an increase in the placebo, and this improvement in prognosis was maintained over the follow-up period. Finally, changes in KidneyIntelX at 1 year were associated with subsequent kidney outcomes, independent of baseline KidneyIntelX scores or treatment with canagliflozin. In sum, these data suggest that KidneyIntelX has clinical utility not only as a prognostic tool but also for longitudinal risk monitoring that may impact on DKD treatment.

Previous studies of KidneyIntelX demonstrated robust risk stratification in a population of patients from 2 medical centers (Mount Sinai and the University of Pennsylvania) in the USA with type 2 diabetes and eGFR $\geq 30 \mathrm{~mL} /$ min or eGFR $\geq 60 \mathrm{~mL} / \mathrm{min}$ with albuminuria [11]. In this prior study by Chan et al. [11], the composite kidney event of RKFD, 40\% sustained decline in eGFR, or kidney failure occurring in $21 \%$ of the population (compared to only $9.9 \%$ in the DKD subcohort in CANVAS) was ascertained through linkage with the electronic health record. None of the patients were treated with the SGLT2i at cohort inception. Some key characteristics were comparable between the prior study population and the CANVAS cohort, including the median age (64 years in CANVAS vs. 63 years in Chan et al. [11]) and median UACR (56 $\mathrm{mg} / \mathrm{g}$ in CANVAS vs. $61 \mathrm{mg} / \mathrm{g}$ in Chan et al. [11]). However, there were many notable differences, including a higher median eGFR $\left(65 \mathrm{~mL} / \mathrm{min} / 1.73 \mathrm{~m}^{2}\right.$ in CANVAS vs. $54 \mathrm{~mL} / \mathrm{min} / 1.73 \mathrm{~m}^{2}$ in Chan et al. [11]), a higher proportion of Caucasians in CANVAS (81\% vs. $33 \%$ in Chan et al. [11]), and higher HbAlc in CANVAS ( $8.2 \%$ vs. $6.9 \%$ in Chan et al. [11]). All patients in CANVAS had the UACR measured at baseline compared to only $62 \%$ in the prior study by Chan et al. [11] (which required imputation of the UACR in the $38 \%$ with a missing UACR). Finally, the processes of care, even in the placebo arm of CANVAS, likely afforded more attention to care than in the observational cohort studied by Chan et al. [11]. Despite the potential differences in the cohorts, covariate structure, and data density, the feature weights for KidneyIntelX that were trained, tested, and validated in the study by Chan et al. [11] were directly applied to CANVAS population with $\mathrm{DKD}$, which yielded very similar performance, including the association with progression for those scoring high risk versus low risk on KidneyIntelX. In the study by Chan et al. [11], KidneyIntelX scored $46 \%$ of the population as low risk as compared to $42 \%$ in CANVAS. The gradient of risk between the high-risk and low-risk KidneyIntelX strata was 6-fold greater in Chan et al. [11] and was 8-fold greater in the CANVAS population. The gradient of risk for high versus low KidneyIntelX risk strata was greater than the gradient of risk for KDIGO very high-risk versus moderate-risk strata (2.5fold greater in Chan et al. [11] and CANVAS). In all, the analyses described above validate that the KidneyIntelX 
risk score is generalizable to broad populations as represented by this external cohort.

While the data from several large randomized controlled trials of SGLT2i are suggestive of relative benefit of SGLT2i treatment across levels of baseline risk and phenotypes of CKD and cardiovascular disease, clinical uptake of SGLT2i has been slow in the underlying patient populations for whom they are indicated [18]. We demonstrated that patients with high KidneyIntelX scores at baseline did derive a slightly greater absolute benefit in terms of kidney function decline over time. While decisions to prescribe medications for DKD are complex and are dependent not only on patient-related factors but also on monetary constraints, pending future data on response to therapy by KidneyIntelX risk stratum, there may be the potential to use the KidneyIntelX test as an aid to improve resource utilization and drive more complex medication regimen decisions that align with current consensus guidelines in those that are at higher risk of progression, including titration of RAAS inhibitors to maximally tolerated doses along with recently approved drugs such as SGLT2is and mineralocorticoid receptor antagonists (e.g., finerenone). Clinician and patient awareness of DKD as well as the underlying risk of progression provided by the KidneyIntelX test may synergize to increase initial acceptance and initiation of new therapies, and in addition, the salutary benefits on the KidneyIntelX risk score may also serve as a positive reinforcement tool to continue compliance with treatment.

Previous studies have demonstrated that canagliflozin reduces plasma TNFR-1, and the changes in TNFR-1 are associated with subsequent eGFR decline [19]. Prior work within the overall CANVAS population [10] demonstrated that canagliflozin reduced the 3 plasma biomarkers that are incorporated into KidneyIntelX compared to the placebo, although the association with future kidney outcomes was quantitatively stronger for changes in plasma TNFR-1 and TNFR-2 despite larger treatment-induced changes in plasma KIM-1 [10]. Clinical implementation of the 3 biomarkers, which represent different pathophysiologic domains (inflammation via the TNFRs and kidney injury via KIM-1) and have orthogonal relationships with traditional kidney metrics [10] as response to biomarkers is quite complex. KidneyIntelX, as a single composite risk score, changed meaningfully in canagliflozin-treated CANVAS participants, and each percentage change in KidneyIntelX predicted risk was associated with future kidney outcomes. While the baseline risk provided by KidneyIntelX was the major prognostic feature of future risk, we were able to demonstrate that transition from a low-risk score at baseline was associated with approximately a 3 -fold increase in risk of future outcomes, and transition from a high-risk score at baseline was associated with a $40 \%$ lower risk of future outcomes. These initial findings demonstrate the potential benefit of longitudinal KidneyIntelX risk scoring. More data will be generated via ongoing prospective studies of KidneyIntelX within observational and clinical trial frameworks.

There are several limitations that need to be considered. First, the findings presented herein are post hoc observational analyses of a randomized controlled trial that cannot be used to infer causality. Second, while the prognostic scoring by KidneyIntelX represents external validation of previous findings [16], the findings of the dynamic changes in KidneyIntelX have not been shown previously and thus need further validation outside of the CANVAS cohort population. Third, although CANVAS was a very large randomized controlled trial of $\geq 4,000$ participants, the proportion with DKD at baseline with available banked samples totaled only 1,325 , and the analytic dataset for the dynamic changes was approximately 1,000 ; thus, some of the findings were limited by a lack of more robust statistical power. Studies in larger populations will help to improve the precision of the point estimates and CIs by each KidneyIntelX risk stratum and their associated changes over time. Finally, while other analyses have demonstrated that KidneyIntelX deployment can result in significant cost savings [20], the degree of cost savings in a population managed with high baseline use of the SGLT2i is yet to be determined.

In conclusion, KidneyIntelX successfully risk-stratified a large multinational external cohort for risk of progression of $\mathrm{DKD}$, with larger differences in the eGFR slope for canagliflozin versus placebo in those with higher versus lower baseline KidneyIntelX scores, as well as compared to KDIGO risk strata. Canagliflozin treatment reduced KidneyIntelX risk scores over time and changes in the KidneyIntelX score from baseline to 1 year predicted future risk of DKD progression. Repeat testing of KidneyIntelX has prognostic and likely clinical utility.

\section{Acknowledgments}

The authors thank all patients and CANVAS investigators for their tremendous support in the trial. CANVAS was sponsored by Janssen Research \& Development, LLC, and was conducted as a collaboration between the sponsor, an academic steering committee, and an academic research organization, George Clinical. Editorial assistance was provided by Cello Health Communications/ MedErgy (Kimberly Dittmar, PhD, and Alaina Mitsch, PhD) and was funded by Janssen Research \& Development, LLC. Cana- 
gliflozin has been developed by Janssen Research \& Development, LLC, in collaboration with Mitsubishi Tanabe Pharma Corporation. The authors also thank the data analytic team at Persistent Systems for the statistical analyses.

\section{Statement of Ethics}

This post hoc study was approved by the Institutional Research Boards of the University of Groningen and the Icahn School of Medicine at Mount Sinai. All individuals provided informed consent before inclusion into the parent study (CANVAS). This study was performed in compliance with the Helsinki Declaration.

\section{Conflict of Interest Statement}

D. Lam received research support from Renalytix and has previously received research support from Novo Nordisk and Insulet Corp. G. Nadkarni has received fees for an advisory board role in Renalytix AI and owns equity in Renalytix. He has received operational funding from Goldfinch Bio and consulting fees from BioVie Inc, AstraZeneca, Reata, and GLG consulting in the past 3 years. He is supported by a career development award from the National Institutes of Health (NIH; K23DK107908) and is also supported by the following NIH grants: R01DK108803, U01HG007278, U01HG009610, and U01DK116100. G. Mosoyan serves as a consultant to Renalytix AI. B. Neal is an employee of the George Institute for Global Health and is supported by an NHM$\mathrm{RC}$ investigator grant. His institution has received fees for his roles in advisory boards, steering committees, or scientific presentations from AstraZeneca, Janssen, Merck, and Mundipharma. K.W. Mahaffey has received research support from Afferent, Amgen, Apple, Inc, AstraZeneca, Cardiva Medical Inc, Daiichi, Ferring, Google (Verily), Johnson \& Johnson, Luitpold, Medtronic, Merck, NIH, Novartis, Sanofi, St. Jude, and Tenax and has served as a consultant (speaker fees for continuing medical education events only) for Abbott, Ablynx, AstraZeneca, Baim Institute, Boehringer Ingelheim, Bristol Myers Squibb, Elsevier, GlaxoSmithKline, Johnson \& Johnson, MedErgy, Medscape, Mitsubishi Tanabe, MyoKardia, NIH, Novartis, Novo Nordisk, Portola, Radiometer, Regeneron, Springer Publishing, and the University of California, San Francisco. N. Rosenthal and M.K. Hansen are employees of Janssen Research \& Development, LLC. H.J.L. Heerspink is sup- ported by a VIDI (917.15.306) grant from the Netherlands Organisation for Scientific Research and has served as a consultant for AbbVie, Astellas, AstraZeneca, Bayer, Boehringer Ingelheim, Chinook, CSL Pharma, Fresenius, Gilead, Janssen, Merck, Mundipharma, Mitsubishi Tanabe, and Retrophin and has received grant support from AbbVie, AstraZeneca, Boehringer Ingelheim, and Janssen. F. Fleming is an executive director of Renalytix AI. S.G. Coca has received fees for advisory boards or steering committee roles for Renalytix, CHF Solutions, Bayer, Boehringer Ingelheim, Takeda, Relypsa (Vifor), Quark, ProKidney, and Akebia in the past 3 years. He owns equity in Renalytix; receives salary and research support from Renalytix, ProKidney, XORTX, and the Renal Research Institute; and receives salary and research support from the following grants from the NIH: U01DK106962, R01DK115562, R01HL85757, R01DK112258, U01OH011326, and R01DK126477.

\section{Funding Sources}

The CANVAS trial and biomarker measurements were funded by Janssen Research \& Development, LLC. The CANVAS trial was done as a collaboration between the funder, an academic steering committee, and an academic research organization (George Clinical). The funder was involved in the study design, data collection, data analysis, data interpretation, and writing of the report.

\section{Author Contributions}

D.L., G.N.N., M.K.H., H.J.L.H., F.F., and S.G.C. were responsible for concept, analytic design, data analysis, interpretation of results, and manuscript preparation. G.M. performed the assays and contributed to the interpretation of results and preparation of the manuscript. B.N., K.W.M, and N.R. contributed to the interpretation of results, provided thoughtful comments, and critically reviewed the manuscript. All the authors contributed to manuscript revisions.

\section{Data Availability Statement}

Clinical data from the CANVAS trial are available in the public domain via the Yale University Open Data Access Project (YODA); http://yoda.yale.edu.

\section{References}

1 Coca SG, Nadkarni GN, Huang Y, Moledina DG, Rao V, Zhang J, et al. Plasma biomarkers and kidney function decline in early and established diabetic kidney disease. J Am Soc Nephrol. 2017;28(9):2786-93.

2 Bhatraju PK, Zelnick LR, Shlipak M, Katz R, Kestenbaum B. Association of soluble TNFR1 concentrations with long-term decline in kidney function: the Multi-Ethnic Study of Atherosclerosis. J Am Soc Nephrol. 2018; 29(11):2713-21.
3 Greenberg JH, Abraham AG, Xu Y, Schelling JR, Feldman HI, Sabbisetti VS, et al. Plasma biomarkers of tubular injury and inflammation are associated with CKD progression in children. J Am Soc Nephrol. 2020;31(5):1067-77.

4 Schrauben SJ, Shou H, Zhang X, Anderson $\mathrm{AH}$, Bonventre JV, Chen J, et al. Association of multiple plasma biomarker concentrations with progression of prevalent diabetic kidney disease: findings from the chronic renal insufficiency cohort (CRIC) study. J Am Soc Nephrol. 2021;32(1):115-26.
5 Tummalapalli L, Nadkarni GN, Coca SG. Biomarkers for predicting outcomes in chronic kidney disease. Curr Opin Nephrol Hypertens. 2016;25(6):480-6.

6 Neuen BL, Ohkuma T, Neal B, Matthews DR, de Zeeuw D, Mahaffey KW, et al. Relative and absolute risk reductions in cardiovascular and kidney outcomes with canagliflozin across KDIGO risk categories: findings from the CANVAS Program. Am J Kidney Dis. 2021; 77(1):23-34 e1. 
7 Neuen BL, Ohkuma T, Neal B, Matthews DR, de Zeeuw D, Mahaffey KW, et al. Cardiovascular and renal outcomes with canagliflozin according to baseline kidney function. Circulation. 2018;138(15):1537-50.

8 Neuen BL, Ohkuma T, Neal B, Matthews DR, de Zeeuw D, Mahaffey KW, et al. Effect of canagliflozin on renal and cardiovascular outcomes across different levels of albuminuria: data from the CANVAS Program. J Am Soc Nephrol. 2019;30(11):2229-42.

9 Neuen BL, Young T, Heerspink HJL, Neal B, Perkovic V, Billot L, et al. SGLT2 inhibitors for the prevention of kidney failure in patients with type 2 diabetes: a systematic review and meta-analysis. Lancet Diabetes Endocrinol. 2019;7(11):845-54.

10 Sen T, Li J, Neuen BL, Neal B, Arnott C, Parikh CR, et al. Effects of the SGLT2 inhibitor canagliflozin on plasma biomarkers TNFR-1, TNFR-2, and KIM-1 in the CANVAS trial. Diabetologia. 2021;64(10):214758.

11 Chan L, Nadkarni GN, Fleming F, McCullough JR, Connolly P, Mosoyan G, et al. Derivation and validation of a machine learning risk score using biomarker and electronic patient data to predict progression of diabetic kidney disease. Diabetologia. 2021;64(7): 1504-15.

12 Neal B, Perkovic V, Mahaffey KW, de Zeeuw D, Fulcher G, Erondu N, et al. Canagliflozin and cardiovascular and renal events in type 2 diabetes. N Engl J Med. 2017;377(7):644-57.

13 Neal B, Perkovic V, Mahaffey KW, Fulcher G, Erondu N, Desai M, et al. Optimizing the analysis strategy for the CANVAS Program: a prespecified plan for the integrated analyses of the CANVAS and CANVAS-R trials. Diabetes Obes Metab. 2017;19(7): 926-35.

14 Oshima M, Neal B, Toyama T, Ohkuma T, Li Q, de Zeeuw D, et al. Different eGFR decline thresholds and renal effects of canagliflozin: data from the CANVAS Program. J Am Soc Nephrol. 2020;31(10):2446-56.

15 Perkovic V, de Zeeuw D, Mahaffey KW, Fulcher G, Erondu N, Shaw W, et al. Canagliflozin and renal outcomes in type 2 diabetes: results from the CANVAS Program randomised clinical trials. Lancet Diabetes Endocrinol. 2018;6(9):691-704.

16 Chan L, Nadkarni GN, Fleming F, McCullough JR, Connolly P, Mosoyan G, et al. Derivation and validation of a machine learning risk score using biomarker and electronic patient data to predict progression of diabetic kidney disease. Diabetologia. 2021;64(7): 1504-15.

17 Chauhan K, Nadkarni GN, Fleming F, McCullough J, He CJ, Quackenbush J, et al. Initial validation of a machine learning-derived prognostic test (KidneyIntelX) integrating biomarkers and electronic health record data to predict longitudinal kidney outcomes. Kidney360. 2020;1(8):731-9.

18 Arnold SV, de Lemos JA, Rosenson RS, Ballantyne CM, Liu Y, Mues KE, et al. Use of guideline-recommended risk reduction strategies among patients with diabetes and atherosclerotic cardiovascular disease. Circulation. 2019;140(7):618-20.

19 Heerspink HJL, Perco P, Mulder S, Leierer J, Hansen MK, Heinzel A, et al. Canagliflozin reduces inflammation and fibrosis biomarkers: a potential mechanism of action for beneficial effects of SGLT2 inhibitors in diabetic kidney disease. Diabetologia. 2019;62(7): 1154-66.

20 Datar M, Burchenal W, Donovan MJ, Coca SG, Wang E, Goss TF. Payer budget impact of an artificial intelligence in vitro diagnostic to modify diabetic kidney disease progression. J Med Econ. 2021;24(1):972-82. 